\title{
Epidermal growth factor receptor analyses in colorectal cancer: A comparison of methods
}

\author{
KAREN-LISE GARM SPINDLER, JAN LINDEBJERG, JENS NEDERBY NIELSEN, DORTE AALUND OLSEN, \\ CLAUS BISGÅRD, IVAN BRANDSLUND and ANDERS JAKOBSEN
}

Danish Colorectal Cancer Group South, University of Southern Denmark, Vejle Hospital, Kabbeltoft 25, 7100 Vejle, Denmark

Received May 23, 2006; Accepted July 17, 2006

\begin{abstract}
EGFR immunohistochemistry (IHC) status is not a reliable predictive marker for response to EGFR-targeted therapies. The present study compares the EGFR status at DNA, RNA and protein level. Blood samples, corresponding normal colon and colorectal cancer tissue were collected from 199 colorectal cancer (CRC) patients. EGFR status was evaluated by FISH analysis, real-time RT-PCR, ELISA and IHC. A polymorphism in the EGFR promoter was evaluated by PCR analysis. The EGFR levels by different methods were mutually compared. Seventy-eight percent of primary tumours and corresponding lymph nodes had equivalent EGFR status (28/34). There was a tendency to higher median protein level (by ELISA) in IHC positive patients compared to IHC negative patients $(\mathrm{p}=0.086)$. The median EGFR gene expression level was significantly lower in tumours than in the normal colon with no difference according to IHC status. No tumours had increased gene copy number by FISH. EGFR Sp1-216 polymorphism analysis showed a tendency for different EGFR tumour protein levels and gene expression levels according to the different genotypes. The results show a poor correlation between EGFR status at DNA, RNA and protein level. The predictive value of a combination of methods needs further evaluation in the clinical setting.
\end{abstract}

\section{Introduction}

Colorectal cancer (CRC) is one of the major cancer diseases in the world (1) and holds a poor prognosis. In recent years the development has indicated that the epidermal growth factor receptor (EGFR) is a valuable target for anticancer therapy and new treatment modalities such as monoclonal antibodies (2-4) and small molecules $(5,6)$ targeting EGFR have shown promising results in the clinical setting. However, only a minor fraction of the patients benefit from these new

Correspondence to: Dr Karen-Lise Garm Spindler, Department of Oncology, Vejle Hospital, Kabbeltoft 25, DK-7100 Vejle, Denmark E-mail:kalgsp@vgs.vejleamt.dk

Key words: colorectal cancer, epidermal growth factor receptor, analysis, methods treatment modalities and predictive markers for outcome are urgently needed.

EGFR is a well-known transmembrane tyrosine kinase receptor and a member of the ErbB family composed of four structurally related transmembrane receptors; EGFR, HER2, HER3 and HER4. The extracellular part of EGFR binds several ligands. Binding of ligand to receptor induces receptor dimerization followed by activation of the tyrosine kinases, which mediates downstream signalling that stimulates the cell cycle pathway and controls cell proliferation. Uncontrolled cell growth, decreased apoptosis, stimulation of angiogenesis and cell proliferation is mediated by dysregulation of the EGFR signalling system. Consequently the EGFR is considered to play a central role in regulation of malignant transformation and tumour growth $(7,8)$. EGFR is present in most epithelial tissue and is overexpressed in various solid tumours among these colorectal cancers (8).

Traditionally the epidermal growth factor receptors have been evaluated by immunohistochemistry (IHC) (9). Overexpression of EGFR has been associated with poor prognosis, shorter survival and increased metastatic ability. In colorectal cancer EGFR overexpression has been reported in $25-82 \%$ (9) and has been shown to predict advanced stage and metastatic potential (10), but the impact on survival remains controversial (11).

A major challenge for all targeted therapies is to identify simple and effective predictive markers allowing a rational treatment selection. So far EGFR testing by IHC has not provided clinicians with a reliable method for selection of patients to EGFR-targeted therapies $(2,12)$. Studies have failed to show any relation between EGFR expression level and the clinical efficacy of cetuximab $(2,13)$. Even tumours with undetectable EGFR levels have responded to cetuximab therapy and response rates in EGFR-negative patients were comparable to those in patients with EGFR-positive tumours (14). These discrepancies have raised several explanations including tumour heterogeneity, poor-sensitivity and lack of standardisation of methodology.

Alternative methods for EGFR expression analysis and the underlying mechanism for EGFR regulation are being actively investigated. Dysregulation of EGFR kinase activity may occur as a consequence of EGFR overexpression, gene amplification or through mutations resulting in constitutive activation. EGFR gene copy number can be detected by FISH analysis as described by Moroni et al (15). However, the role of gene amplification and gene copy number assessment in 
clinical settings needs further evaluation. Measurement of gene expression levels has been evaluated by real-time RT-PCR (16) and holds promising potential as a predictive marker in cetuximab therapy. EGFR protein quantification is assessable by IHC and enzyme-linked immunosorbent assay (ELISA). Apart from that on IHC, the literature on these methods is scarce regarding CRC.

Recently gene polymorphisms relating to the EGFR signalling system have been described. A functional polymorphism in the Sp1 binding site of the EGFR promoter region has been identified and reported to influence the gene expression level of EGFR in cell lines (17). There are no clinical data on the possible relationship between this Sp1216 EGFR polymorphism and EGFR gene-expression levels.

The aim of the present study was to compare different methods for EGFR-analysis in CRC. We investigated the receptor status in colorectal adenocarcinoma tissue as well as in normal colon tissue and blood, with the purpose of comparing the EGFR status at DNA, RNA and protein level.

\section{Patients and methods}

Patients. The study included 199 patients with CRC during the period of December 2003 to July 2005. All patients underwent surgical resection for adenocarcinomas of the colon or rectum at Department of Surgery, Vejle Hospital, Denmark. Disease extension was classified according to the TNM system. Samples of blood, colorectal tumours and normal colon were collected at surgery after obtaining informed consent from the patients. The study was approved by the Regional Ethics Committee of Vejle and Funen Counties according to Danish law.

Sampling. Fresh tissue from tumour and normal colon was frozen in Tissuetek O.C.TTM compound (SAKRUA,) and prepared for protein analysis. Additional tissue samples were stored at $-20^{\circ} \mathrm{C}$ in RNA-later (Qiagen, CA, USA). Further samples and corresponding lymph node metastases were formalin-fixed and paraffin-embedded according to standard procedure.

Immunohistochemistry. Colorectal specimens were immersed in $4 \%$ buffered neutral formalin and fixed for $24 \mathrm{~h}$. Paraffinembedding was performed according to standard procedures. Sections of $4-\mu \mathrm{m}$ were mounted on coated slides and allowed to dry for $30 \mathrm{~min}$ at $60^{\circ} \mathrm{C}$ and overnight at $37^{\circ} \mathrm{C}$. All sections were stained within $24 \mathrm{~h}$ of embedding. The slides were deparafinized in ESTISOL 220 (Esti Chem) and rehydrated in graded alcohol solutions. Endogenous peroxidase was blocked with $3 \%$ hydrogen peroxide. Proteolytic antigen retrieval was performed using $0.1 \%$ protease at room temperature (RT) for $20 \mathrm{~min}$. Slides were incubated in primary mouse anti-EGFR Mab (clone H-11, Dako Corporation, Carpinteria, USA) for $30 \mathrm{~min}$ at RT. Visualisation of the reaction was performed using ENVISION + DAB (Dako Cytomation-DK) followed by counterstaining with haematoxylin. Staining was performed manually.

Evaluation of EGFR IHC. Evaluation was independently performed by two investigators (Karen-Lise Garm Spindler and Jan Lindebjerg). EGFR positivity was defined according to Dako guidelines, any membrane staining above background level was considered positive. Tumours were graded with regard to intensity and amount of membrane staining. A score of staining intensity was assigned as follows: $1+$, weak; $2+$, moderate; and $3+$, strong membrane staining. The tumour was defined positive if $\geq 1 \%$ of the cells had membranous staining for EGFR according to the Dako guidelines. A score was defined according to the percentage of positively stained tumour cells as follows: $0,<1 \% ; 1,1-10 \% ; 2,10-25 \% ; 3$, $25-50 \% ; 4,>50 \%$.

\section{Quantitative EGFR ELISA}

Extraction of proteins. Tissue samples of $10-50 \mathrm{mg}$ were homogenised at $4^{\circ} \mathrm{C}$ by an ultra-turrax system (Ika, Germany) with $10 \mathrm{Vol}(\mathrm{w} / \mathrm{v})$ buffer $(20 \mathrm{mM}$ Tris- $\mathrm{HCl}, 150 \mathrm{mM} \mathrm{NaCl}$, pH 7.4, 1 mM EDTA, 10\% glycerol, protease inhibitor cocktail (cat. no. P8340, Sigma-Aldrich, USA). After homogenisation, Triton X-100 was added to the samples to a final concentration of $1 \%(\mathrm{v} / \mathrm{v})$. The lysates were mixed and incubated for $30 \mathrm{~min}$ at $4^{\circ} \mathrm{C}$ followed by centrifugation at $16000 \mathrm{x} \mathrm{g}$ for $10 \mathrm{~min}$ at $4^{\circ} \mathrm{C}$. The supernatants were recovered and the protein concentration was determined using the bicinchorinic acid protein assay (Pierce, USA).

EGFR ELISA. A commercially available enzyme-linked immunosorbent assay (Oncogene Science, USA) was used to quantify EGFR in colon cancer tissue and autologous reference tissue. Tissue extracts were adjusted in sample diluent to a final protein concentration of $\sim 50 \mu \mathrm{g} / \mathrm{ml}$. Diluted tissue samples along with standards and controls (Oncogene Science) were added to a 96-well microtiter plate coated with a mouse monoclonal anti-(EGFR) antibody and incubated for $1.5 \mathrm{~h}$ at $37^{\circ} \mathrm{C}$. After this incubation step plates were washed and incubated with an alkaline phosphatase-labelled mouse monoclonal anti-(EGFR) antibody for $30 \mathrm{~min}$ at RT. Enzymatic reactions were carried out at RT by adding BluePhos substrate and the reaction was stopped after $60 \mathrm{~min}$ by the addition of stop solution. Colour development was measured at $650 \mathrm{~nm}$ by using an automated plate reader (Vmax, Molecular Devices, USA) and the EGFR concentration of the unknown samples was estimated from the standard curve. All samples were analysed in duplicate and the average of the two was recorded. The interassay and intraassay coefficients of variation were $<10 \%$.

\section{Relative gene expression analysis of EGFR}

RNA isolation and cDNA synthesis. Total RNA was isolated using an RNeasy kit from Qiagen according to the manufacturer's instructions. Isolated RNA was quantitated by Spectrophotometry (Ebbendorf, Hamburg, Germany) and cDNA synthesis was performed using M-MLV RT (Invitrogen) as previously described (18).

Real-time PCR quantification of $m R N A$ expression. Following RNA isolation and cDNA synthesis real-time fluorescence PCR was performed for t-EGFR and $\beta$-actin using an assay from Applied Biosystems (Hs01076088_m1 4310881E respectively) on an ABI PRISM HT 7900 sequence detection system, TaqMan (Perkin-Elmer Applied Biosystem, Foster 
Table I. Primers and probes for Sp1-216 G/T gene polymorphism analysis.

\begin{tabular}{lll}
\hline Probes and primers & \multicolumn{1}{c}{ Sequence } & \multicolumn{1}{c}{ System } \\
\hline EGFR-Sp1G probe & 6FAM - AGC AGC CTC CGC C & ABI PRISM 7900 HT \\
EGFR-Sp1T probe & VIC - AGC AGC CTC CTC C & ABI PRISM 7900 HT \\
EGFR-Sp1 forward primer & CGT CCG GGC AGC CC & ABI PRISM 7900 HT \\
EGFR-Sp1 reverse primer & GGC GCT CAC ACC GTG C & ABI PRISM 7900 HT \\
Forward sequencing primer & GGT CTC CTC CTC CTC CTC GCA & ABI 3100 \\
Reverse sequencing primer & TTG TGG CGT TGG CGG CGA & ABI 3100 \\
\hline
\end{tabular}

City, CA, USA). The housekeeping gene $\beta$-actin was used as a denominator for standardization. The PCR mixture and cycling conditions were conducted according to the manufacturer's instructions. The relative gene expression was determined based on the threshold cycles of EGFR and the internal standard B-actin. Quantification was performed as previously published using a standard curve model (19). The line of the EGFR standard curve was $y=-3.3728 x+35.098$ and the linear regression coefficient $\mathrm{R}^{2}=0.9948$. The line and regression coefficient of $B$-actin standard curve were $y=3.423 X+29.141$ and $R^{2}=0.9992$ respectively. Positive controls (samples of known value) and negative controls (samples without cDNA) were performed in parallel for each PCR experiment ensuring equivalent assay conditions. Quantifications of mRNA were carried out in triplicates.

Sp1-216 G/T EGFR polymorphism analysis. Genomic DNA was isolated from whole blood as previously described (19). Polymerase chain reaction (PCR) analysis of Sp1 gene polymorphism in the EGFR promoter was performed using the ABI PRISM 7900 HT sequence detection system (PerkinElmer Applied Biosystem). The results were verified by sequencing on an ABI 3100 sequence detection system. Table I shows primers and probes.

FISH analysis. The tumour samples were paraffin-embedded and formalin-fixed according to standard procedures. FISH was performed on $3-\mu \mathrm{m}$ sections using the FISH accessory kit from Dako Cytomation-DK together with a fluorescently labelled DNA probe set, (LSI EGFR SpectrumOrange/CEP 7 SpectrumGreen Probe) from Vysis, Inc. (Downers Grove, IL, USA). Staining procedures were performed according to the manufacturer's instructions. Tumour samples were examined with a Zeiss Axio Imager fluoroscense microscope. Evaluation was made by a single trained pathologist (Jan Lindebjerg) who was blinded to patients' data.

Evaluation of FISH analysis. In each tumour sample signals were counted in a total of 100 non-overlapping tumour-cell nuclei. The mean signal number of the EGFR gene as well as CEP7 was calculated and the EGFR gene/CEP7 ratio determined.

Statistical analyses. The correlation between EGFR status and various clinicopathological parameters was determined by the Student's t-test, Fisher's exact test or Wilcoxon ranksum test for difference in medians when appropriate. The inter- and intra-observer reproducibility of assessments of the IHC staining was tested by calculating Cohen's $\kappa$. The relationship between EGFR immunostaining in primary tumours and metastasis was evaluated by Fisher's exact test. Linear regression analysis was used to describe the correlation between EGFR ELISA protein and gene expression levels in tumour and normal colon. Fisher's exact test was used to evaluate the proportions of IHC positive and negative tumours in the different groups of Sp1-216 genotype. P-values $\leq 0.05$ were considered significant and all statistics were carried out using the NCSS statistical software (NCSS Statistical Software, UT 84037, USA).

\section{Results}

Patient characteristics are shown in Table II. The median age was 71 years (range 41-91 years). All tumours were histopathologically confirmed adenocarcinomas of the colon or rectum.

$I H C$. One hundred and ninety-three tumours were available for EGFR IHC staining and 51\% (99/193) of tumours were positive. There was no correlation between EGFR score and clinicopathological parameters in terms of age, gender, tumour location and TNM category. Thirty-four patients had corresponding lymph node metastasis available for EGFR staining. One third $(5 / 15)$ of patients with EGFR-positive tumours had EGFR-negative lymph node metastasis whereas lymph node positive metastasis was found in only 5\% (1/19) of patients with negative tumours (Table III). Primary tumours and lymph node metastasis had equivalent EGFR expression in $78 \%(28 / 34)$ of observations.

EGFR protein ELISA. EGFR protein ELISA was performed on 94 paired tumour and normal colon tissue samples. Data are presented in Table IV. There was no difference between the median protein levels of the two groups. Linear regression analysis showed a weak correlation between protein levels in normal tissue and tumours $\left(0.22, \mathrm{R}^{2}=0.05\right)$. The median EGFR protein level in the IHC negative group was $13 \mathrm{ng} / \mathrm{mg}$ compared to $15.3 \mathrm{ng} / \mathrm{mg}$ in patients with IHC positive tumours. The difference was not significant $(\mathrm{p}=0.086)$. EGFR protein level did not correlate with IHC score or intensity (data not shown).

EGFR gene expression analysis. EGFR gene expression analysis was performed on 82 corresponding primary tumours 
Table II. Additional clinicopathological parametres (NS, not significant).

\begin{tabular}{lcc}
\hline Parameter & $\begin{array}{c}\text { Number } \\
\mathrm{N}=199(\%)\end{array}$ & $\begin{array}{c}\text { Correlation } \\
\text { to IHC }\end{array}$ \\
\hline
\end{tabular}

\begin{tabular}{lrr}
\hline Gender & & NS \\
Male & $107(54)$ & \\
Female & $92(46)$ &
\end{tabular}

Topography

Right colon

Left colon

Rectum

$79(40)$

Right and left colon

4 (2)

T category

1

2

$34(17)$

4

Other

$1(0.5)$

N category

0

1

2

3

Unknown

$106(53)$

34 (17)

$36(18)$

$22(11)$

$1(0.5)$

M category

0

$145(73)$

$39(20)$

Unknown

Available for IHC of primary

tumour

EGFR-positive

EGFR-negative

Available for IHC of meta-

stasis

EGFR-positive

EGFR-negative

Available for gene-expression analysis of primary tumours and normal colon tissue

Available for protein ELISA analysis of primary tumour and normal colon tissue

Available for FISH analysis

of primary tumours

Available for Sp1 analysis

NS

NS
Table III. EGFR IHC status in primary tumours and corresponding lymph node metastasis $(\mathrm{p}=0.00015$, Fisher's exact test).

\begin{tabular}{lccc}
\hline & $\begin{array}{c}\text { Metastasis } \\
\text { EGFR-positive }\end{array}$ & $\begin{array}{c}\text { Metastasis } \\
\text { EGFR-negative }\end{array}$ & Total \\
\hline $\begin{array}{l}\text { Primary tumour } \\
\text { EGFR-positive }\end{array}$ & 10 & 5 & 15 \\
$\begin{array}{l}\text { Primary tumour } \\
\text { EGFR-negative }\end{array}$ & 1 & 18 & 19 \\
Total & 11 & 23 & 34 \\
\hline
\end{tabular}

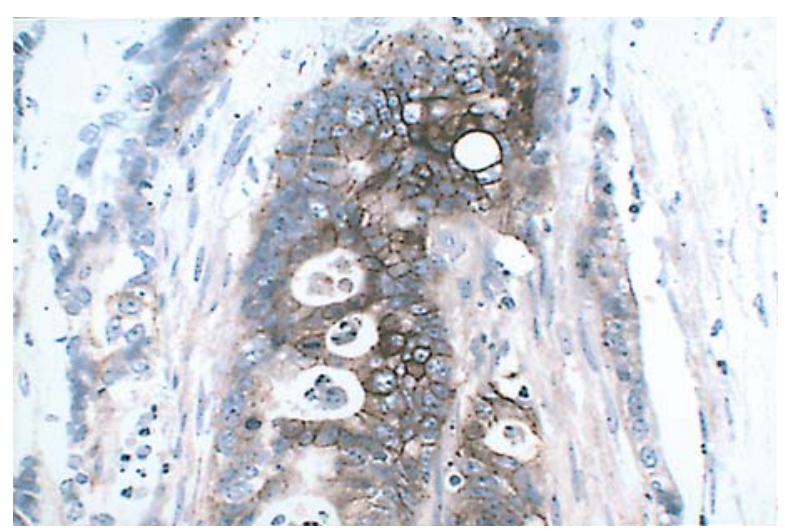

Figure 1. Immunohistochemical staining of EGFR in colorectal adenocarcinoma with intensity score $3+$.

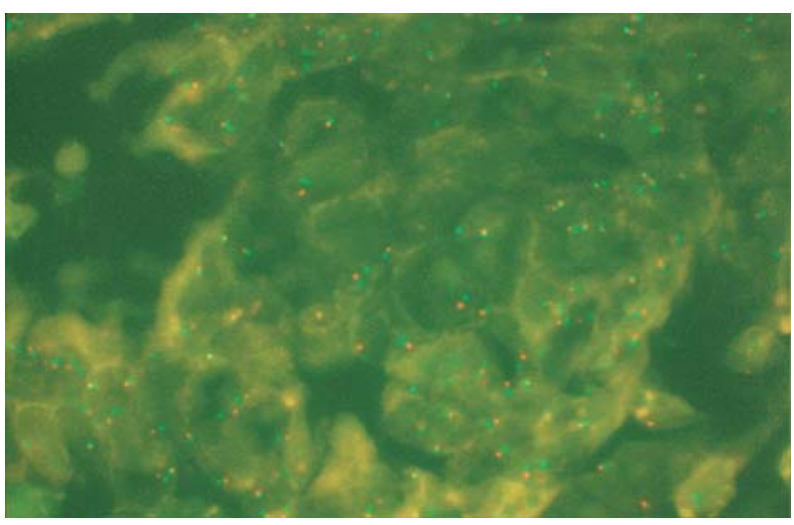

Figure 2. FISH analysis of EGFR in CRC. Red dots, EGFR gene; green dots, CEP7.

and normal colon tissue. The median EGFR level was 1.05 $(0.98-1.23,95 \% \mathrm{Cl}$, range $0.49-2.2)$ in the normal colon compared to $0.7(0.63-0.85,95 \% \mathrm{Cl}$, range $0.18-1.49)$ in the colorectal tumours (Fig. 3). The difference was significant $\left(\mathrm{p}=10^{-6}\right)$. There were no significant differences in EGFR gene expression levels between IHC positive and IHC negative tumours. Data are presented in Table IV. EGFR protein analysis by ELISA did not reflect the gene expression level of EGFR with no correlation between tumour protein level and 
Table IV. Quantitative analysis of EGFR in tumour tissue and normal colon.

\begin{tabular}{|c|c|c|c|c|}
\hline Method & Range & Median & $95 \% \mathrm{CL}$ & p-value \\
\hline EGFR gene-expression & (qEGFR/qBA) & (qEGFR/qBA) & & \\
\hline Normal colon & $0.49-2.20$ & 1.05 & $0.98-1.23$ & $10^{-6}$ \\
\hline Tumour overall & $0.18-1.49$ & 0.70 & $0.63-0.85$ & \\
\hline IHC positive & $0.26-1.49$ & 0.70 & $0.63-0.85$ & 0.92 \\
\hline IHC negative & $0.20-1.45$ & 0.71 & $0.53-0.89$ & \\
\hline EGFR protein ELISA & $(\mathrm{ng} / \mathrm{mg})$ & (ng/mg) & & \\
\hline Normal colon & $2.5-36.3$ & 14.35 & 12.4-15.9 & \\
\hline Tumour overall & $2.9-49.6$ & 13.65 & $12.2-15.6$ & 0.98 \\
\hline IHC positive & $5.6-49.6$ & 15.30 & 12.4-17.9 & \\
\hline IHC negative & $2.9-39.9$ & 13.20 & $9.5-15.9$ & 0.086 \\
\hline \multicolumn{5}{|c|}{ EGFR gene-copy number by FISH } \\
\hline Tumour overall & $127-198$ & 151 & $145-154$ & \\
\hline IHC positive & & 151 & $143-157$ & \\
\hline IHC negative & & 150 & $142-154$ & NS \\
\hline \multicolumn{5}{|l|}{ EGFR gene/CEP7 ratio } \\
\hline Tumour overall & $0.928-1.151$ & 0.9935 & $0.9833-1.007$ & \\
\hline IHC positive & & 0.9833 & $0.60-1.007$ & \\
\hline IHC negative & & 1.0 & $0.988-1.026$ & NS \\
\hline
\end{tabular}

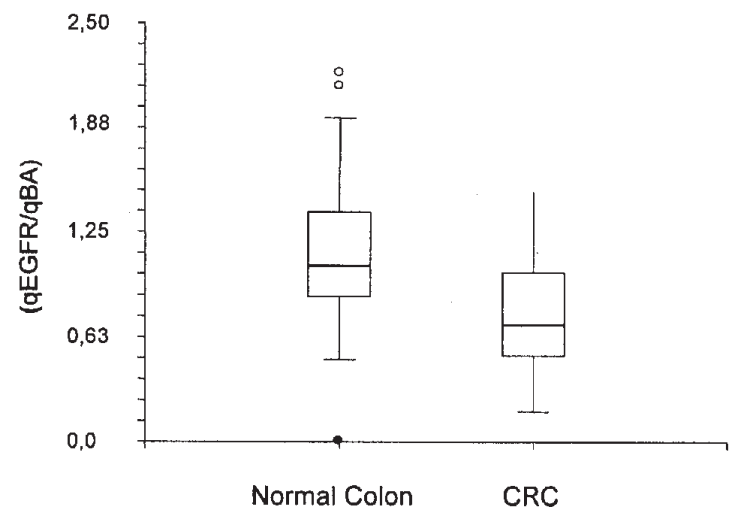

Figure 3. Boxplot of EGFR gene expression levels in normal colon and tumour. Vertical, EGFR gene expression level (qEGFR/qBA); horizontal, normal colon tissue and adenocarcinomas.

EGFR gene expression. Furthermore EGFR gene expression was not correlated to clinicopathological parameters or IHC score.

Spl polymorphism. Seventy-nine patients were available for $\mathrm{Sp} 1$ analysis. Forty-six percent (36/79) of the patients had the GG genotype, 46\% (36/79) and 9\% (7/79) were GT and TT respectively. There were no significant differences in median EGFR tumour gene expression levels according to Sp1-216 EGFR genotype but there was a tendency of TT genotype corresponding to a lower level than the GG groups $(p=0.067)$ (Fig. 4) and that $\mathrm{T}$ containing variants had lower EGFR

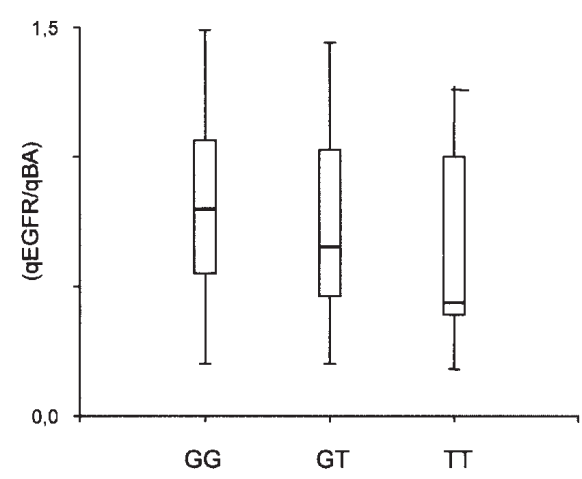

Figure 4. EGFR gene expression (qEGFR/qBA) according to EGFR Sp1-216. Vertical, EGFR gene expression level (qEGFR/qBA); horizontal, EGFR Sp1-216 genotype.

tumour protein levels than GG variants $(p=0.08)$. There were no differences between the $\mathrm{Sp} 1$ groups according to IHC score or clinicopathological parameters.

EGFR gene copy number. Sixty tumours were evaluated by FISH analysis. The median EGFR gene copy number was 151 (range 127-198) with no difference between IHC positive and IHC negative tumours (150 and 151 respectively). Results are shown in Table IV. The median EGFR gene/CEP7 ratio was 0.99 with a range between 0.929 and 1.15 , indicating no gene amplification. When using the definition for balanced ratio as described by Sauer et al (20) (EGFR gene/CEP7 ratio between 0.8 and 1.2) all tumours in this study were balanced. 


\section{Discussion}

There is no consensus with regard to the clinical importance of EGFR evaluation by immunohistochemistry $(21,22)$. Concerning prognosis the current results are contradictory. Furthermore there is no clear indication that EGFR testing by immunohistochemistry can be used to predict the response to anti-EGFR therapies $(2,13,14)$ as described previously.

The apparent discrepancies concerning EGFR evaluation by IHC in CRC have raised several hypotheses. Methodological problems are of major importance. Inconsistent interpretation and use of different antibodies contribute to the problem as discussed by Goldstein and Armin (23). The presence of both high- and low-affinity EGF receptors might be important and Atkins and colleges have shown that the commonly used EGFRpharmDx kit depends on fixative type and storage time and it is not suitable for testing archival tissue in CRC (24). In addition multiple different scoring systems have been used according to the different studies. In the present study we used anti-EGFR antibodies from Dako and tissues sections were cut and stained within the appropriate time. A standardized scoring system according to the manufacturer's guideline was used.

The EGFR IHC expression rate shows considerable variability in the literature. Scartozzi et al (12) found that $53 \%$ of the primary tumours were positive when defining cut-off for EGFR positivity by $\geq 1 \%$, which is similar to data presented here. On the other hand this is in disagreement with other studies (11). The present data showed that IHC score did not correlate to EGFR levels as measured by any of the methods presented here. These data point out the variability of EGFR detection by IHC.

Most studies address EGFR in primary tumours, which might be biologically different from the metastatic lesions. Scartozzi et al found that $36 \%$ of EGFR-positive tumours had EGFR-negative metastases and $15 \%$ of the EGFR-negative tumours had EGFR-positive metastases (12). McKay and colleagues (10) showed that only $40.5 \%$ of paired samples of tumour and metastases had equivalent EGFR expression and Bralet et al found that EGFR expression was positive in $73 \%$ of corresponding primary tumours and metastasis. In the present study we analysed 34 corresponding primary tumours and lymph node metastasis and found equivalent EGFR expression in $78 \%$ of the paired samples (Table III). These data support the idea that IHC staining of primary tumours for treatment selection to metastatic disease might be insufficient. The lack of correlation between EGFR status in primary tumour and metastatic sites could be related to possible genetic changes, tumour heterogeneity as well as methodological problems. Still if a standardised reproducible assay was developed, the question is whether it would provide the clinicians with reliable tools for treatment selections in these settings.

The diverging results of IHC have led to the consequence that several alternative methods have been proposed for evaluation of the biological activity of EGFR. The present study evaluated the relationship between EGFR at the DNA, RNA and protein levels in tumour and normal tissue. The results of this study underline the complexity of the EGFR signalling system.
EGFR can be addressed at the DNA level in several ways of which gene copy number assessment by FISH analysis has shown promising but inconsistent results. Recently Ooi et al found that $58 \%$ of IHC positive colorectal cancers were amplified, and showed a correlation between IHC and FISH, but not between primary tumour and metastasis (25). On the contrary Sauer and colleagues showed that all tumours with gene copy loss were IHC positive suggesting that EGFR gene copy loss is a surrogate marker for EGFR mutation/deletion (20). Moroni et al report that increased EGFR gene copy number may be used as predictor for response to cetuximab treatment (15). The study included 31 patients receiving either cetuximab monotherapy $(n=12)$, cetuximab + irinotecan $(n=9)$ or panitumumab $(n=10)$. Eight out of 9 responders had an increased gene copy number compared to $1 / 20$ of the nonresponders. Consequently assessment of gene copy number was suggested as a potential predictive marker for response in this setting. In the present study all tumours were balanced with respect to EGFR gene copy/CEP7 ratio with a very narrow range, which is in disagreement with the above mentioned literature. The data presented here indicate that gene amplification in CRC is rare and its application as a predictive marker is dubious.

Gene expression analysis by real-time RT-PCR has been evaluated as a molecular determinant of cetuximab efficacy in colorectal cancer by Vallböhmer and colleagues (16). Thirty-nine patients were included in the study. There was no association between IHC and gene expression of EGFR, which is in concordance with the data presented here. Furthermore Vallböhmer et al reported no association between mRNA EGFR and response but found that a low mRNA EGFR level was associated with longer survival compared to patients with high mRNA levels. We did not find any association between protein levels, $\mathrm{Sp1-216}$ polymorphism nor gene copy number and gene expression levels, underlining the complexity of EGFR testing. The present data showed a significantly higher EGFR expression in normal colon tissue compared to colon tumours. These findings are supported by data from EGFR gene expression analysis in prostate cancer patients (26) and challenge the common opinion of EGFR overexpression as a main contributor to EGFR dysregulation. Obviously the issue needs further evaluation in prospective trials.

EGFR protein level was evaluated by traditional IHC and ELISA on fresh frozen tissue. The literature on EGFR ELISA on fresh frozen tissue is limited. It was our aim to investigate if EGFR quantification by ELISA correlated to the IHC score. EGFR ELISA protein level did not reflect IHC scoring intensity and did not correlate to gene expression levels. The role of ELISA quantification of EGFR as a supplement to other methods needs further investigation.

The present study is the first evaluation of the possible relationship between EGFR Sp1-216 polymorphism and EGFR gene expression in CRC. Forty-seven percent of patients had GG genotype, $43 \%$ and $9 \%$ were GT and TT respectively. This distribution of genotype in the population is in concordance with the data produced by Liu et al who furthermore reported that cell lines with the TT variant had low gene expression levels of EGFR (17). The present study showed a tendency of the TT genotype corresponding to a lower level than the GG 
group. Furthermore T containing variants showed a tendency of lower EGFR ELISA protein levels than GG variants. Obviously the low number of observations in the TT group should be considered when interpreting these data. Liu and colleagues suggested that $-216 \mathrm{G} / \mathrm{T}$ polymorphisms might contribute to the inter-individual variability in EGFR expression and response to EGFR targeted therapies. Analysis of EGFR Sp1-216 G/T polymorphism is a reliable and easy method. Therefore it could be a good candidate for a predictive marker but more data from clinical trials are needed.

In conclusion the results of the present study show a poor correlation between EGFR status as measured by different methods at DNA, RNA and protein level. Our results underline the complexity of the EGFR regulation. Therefore a comparison of different methods should be taken with caution. Prediction of treatment effect can probably not rely on a single method. A further step forward would be to investigate the possible value of a combination of different methods in clinically wellcharacterised patient populations.

\section{Acknowledgements}

We thank Lone Frischknecht, Birgit Roed Sørensen and Anette Buhl for their technical assistance and Kirsten Birgitte Hjermitslev for proofreading. The study was supported by grants from Vejle County.

\section{References}

1. Parkin DM, Bray F, Ferlay J and Pisani P: Estimating the world cancer burden: Globocan 2000. Int J Cancer 94: 153-156, 2001.

2. Cunningham D, Humblet Y, Siena S, et al: Cetuximab monotherapy and cetuximab plus irinotecan in irinotecan-refractory metastatic colorectal cancer. N Engl J Med 351: 337-345, 2004.

3. Tyagi P: Recent results and ongoing trials with panitumumab (ABX-EGF), a fully human anti-epidermal growth factor receptor antibody, in metastatic colorectal cancer. Clin Colorectal Cancer 5: 21-23, 2005

4. Calvo E and Rowinsky EK: Clinical experience with monoclonal antibodies to epidermal growth factor receptor. Curr Oncol Rep 7: $96-103,2005$.

5. Blanke CD: Gefitinib in colorectal cancer: if wishes were horses. J Clin Oncol 23: 5446-5449, 2005.

6. Shepherd FA, Rodrigues PJ, Ciuleanu T, et al: Erlotinib in previously treated non-small-cell lung cancer. N Engl J Med 353: 123-132, 2005.

7. Marmor MD, Skaria KB and Yarden Y: Signal transduction and oncogenesis by ErbB/HER receptors. Int J Radiat Oncol Biol Phys 58: 903-913, 2004.

8. Yarden Y and Sliwkowski MX: Untangling the ErbB signalling network. Nat Rev Mol Cell Biol 2: 127-137, 2001.

9. Spaulding DC and Spaulding BO: Epidermal growth factor receptor expression and measurement in solid tumors. Semin Oncol 29: 45-54, 2002.
10. McKay JA, Murray LJ, Curran S, et al: Evaluation of the epidermal growth factor receptor (EGFR) in colorectal tumours and lymph node metastases. Eur J Cancer 38: 2258-2264, 2002.

11. Spano JP, Lagorce C, Atlan D, et al: Impact of EGFR expression on colorectal cancer patient prognosis and survival. Ann Oncol 16: 102-108, 2005.

12. Scartozzi M, Bearzi I, Berardi R, Mandolesi A, Fabris G and Cascinu S: Epidermal growth factor receptor (EGFR) status in primary colorectal tumors does not correlate with EGFR expression in related metastatic sites: implications for treatment with EGFR-targeted monoclonal antibodies. J Clin Oncol 22: 4772-4778, 2004

13. Saltz LB, Meropol NJ, Loehrer PJ Sr, Needle MN, Kopit J and Mayer RJ: Phase II trial of cetuximab in patients with refractory colorectal cancer that expresses the epidermal growth factor receptor. J Clin Oncol 22: 1201-1208, 2004

14. Chung KY, Shia J, Kemeny NE, et al: Cetuximab shows activity in colorectal cancer patients with tumors that do not express the epidermal growth factor receptor by immunohistochemistry. J Clin Oncol 23: 1803-1810, 2005.

15. Moroni M, Veronese S, Benvenuti S, et al: Gene copy number for epidermal growth factor receptor (EGFR) and clinical response to antiEGFR treatment in colorectal cancer: a cohort study. Lancet Oncol 6: 279-286, 2005.

16. Vallböhmer D, Zhang W, Gordon M, et al: Molecular determinants of cetuximab efficacy. J Clin Oncol 23: 3536-3544, 2005.

17. Liu W, Innocenti $\mathrm{F}, \mathrm{Wu} \mathrm{MH}$, et al: A functional common polymorphism in a Sp1 recognition site of the epidermal growth factor receptor gene promoter. Cancer Res 65: 46-53, 2005.

18. Lindebjerg J, Nielsen JN, Hoeffding LD, Bisgaard C, Brandslund I and Jakobsen A: Expression of thymidylate synthase in primary colorectal adenocarcinoma. Appl Immunohistochem Mol Morphol 14: 37-41, 2006.

19. Jakobsen A, Nielsen JN, Gyldenkerne N and Lindeberg J: Thymidylate synthase and methylenetetrahydrofolate reductase gene polymorphism in normal tissue as predictors of fluorouracil sensitivity. J Clin Oncol 23: 1365-1369, 2005.

20. Sauer T, Guren MG, Noren T and Dueland S: Demonstration of EGFR gene copy loss in colorectal carcinomas by fluorescence in situ hybridization (FISH): a surrogate marker for sensitivity to specific anti-EGFR therapy? Histopathology 47: 560-564, 2005.

21. Nicholson RI, Gee JM and Harper ME: EGFR and cancer prognosis. Eur J Cancer 37: 9-15, 2001.

22. Steele RJ, Kelly P, Ellul B and Eremin O: Epidermal growth factor receptor expression in colorectal cancer. Br J Surg 77: 1352-1354, 1990.

23. Goldstein NS and Armin M: Epidermal growth factor receptor immunohistochemical reactivity in patients with American Joint Committee on Cancer Stage IV colon adenocarcinoma: implications for a standardized scoring system. Cancer 92: 1331-1346, 2001.

24. Atkins D, Reiffen KA, Tegtmeier CL, et al: Immunohistochemical detection of EGFR in paraffin-embedded tumor tissues: variation in staining intensity due to choice of fixative and storage time of tissue sections. J Histochem Cytochem 52: 893-901, 2004.

25. Ooi A, Takehana T, Li X, et al: Protein overexpression and gene amplification of HER-2 and EGFR in colorectal cancers: an immunohistochemical and fluorescent in situ hybridization study. Mod Pathol 17: 895-904, 2004.

26. Turkeri LN, Sakr WA, Wykes SM, et al: Comparative analysis of epidermal growth factor receptor gene expression and protein product in benign, premalignant, and malignant prostate tissue. Prostate 25: 199-205, 1994 